\title{
Die Ideologie der Sharing Economy
}

\author{
Sebastian Jürss \& Nils S. Borchers
}

Keywords: Ideologie, Sharing Economy, Solutionismus, digitaler Kapitalismus

\begin{abstract}
Der Beitrag untersucht die ideologische Grundlage des digitalen Kapitalismus, die als Solutionismus verstanden wird. Am Beispiel der Sharing Economy als einer im Zuge der fortschreitenden Digitalisierung entstandenen Ökonomie vollziehen wir diese ideologische Grundlage exemplarisch nach. Hierzu arbeiten wir mittels einer narrativen Diskursanalyse die Ideologiearchitektur aus den Manifesten sowie Anti-Manifesten der Sharing Economy heraus.
\end{abstract}

Jürss, Sebastian, und Nils S. Borchers. 2019. „Die Ideologie der Sharing Economy“. In Ideologie, Kritik, Öffentlichkeit. Verhandlungen des Netzwerks Kritische Kommunikationswissenschaft, herausgegeben von Uwe Krüger und Sebastian Sevignani, 176199. Universität Leipzig. DOI: 10.36730/ideologiekritik.2019.9

Sebastian Jürss | Universität Bremen | s.juerss@uni-bremen.de Nils S. Borchers | Universität Leipzig | nils.borchers@uni-leipzig.de 


\section{Einleitung}

Die Sharing Economy wird in der Forschung wie auch außerhalb des akademischen Diskurses häufig als eine Folge der globalen Finanzkrise in den Jahren 2007/08 gesehen (Nachtwey und Seidl 2017, 3). Ungeachtet der enormen Breite des Phänomens und diversen Akzentuierungen (wie platform economy, gig economy) zeichnet sich ab, dass im öffentlichen Diskurs mit der Sharing Economy vor allem die Sharing-Plattformen als Repräsentation der Sharing Economy gesehen werden. Damit geht eine gewisse Verschiebung weg von dem eigentlichen Zweck des Teilens (wie Mitfahrgelegenheiten, Essen teilen o. ä.) hin zur Plattformlogik als tragendem Prinzip einher. Ein erheblicher Teil des regelrechten Hypes um die Sharing Economy dürfte den großen Plattformen wie etwa Airbnb und Uber zuzurechnen sein. Diese repräsentieren aber nur einen Teil des gesamten Spektrums. Trotzdem bzw. gerade auf Grund ihrer Erfolgsgeschichten hat sich ein öffentlicher Diskurs entwickelt, der sich in der Anfangsphase zunächst an dem Potenzial der Sharing Economy und ihrer Plattformen abgearbeitet hat, im Laufe der Zeit aber auch durch kritischere Stimmen, vor allem angesichts sich verfestigender Konsequenzen wie der Transformation von Innenstadtquartieren durch Airbnb, erweitert hat.

Ziel unseres Beitrags ist es, die Sharing Economy als Teil der neu entstandenen digitalisierten Ökonomien (etwa Nachtwey und Seidl 2017) und damit emblematisch für deren Ideologie zu verstehen. Diese Ideologie wollen wir anschließend einer (ideologie-)kritischen Diskussion unterziehen. Eine solche Diskussion ist erstens notwendig, um die (Nicht)Transformation der gegenwärtigen kapitalistischen Gesellschaft und die Rolle der Digitalisierung zu verstehen (als neuer Geist des Kapitalismus, sensu Boltanski und Chiapello 2003). Zweitens ist sie notwendig, um die zwangsläufig leerlaufenden Versprechen einer Venture-Capital-geförderten Sharing Economy zu identifizieren und nicht, wie Befürworter*innen der Sharing Economy gerne behaupten (etwa Botsman und Rogers 2011), als eine gesellschaftliche Entwicklung hin zu einer "sharing society“ aufzusitzen. Unser Ziel ist es explizit nicht, eine ausführliche Exegese der bestehenden Literatur zu Ideologie und Ideologiekritik zu betreiben, sondern mit Hilfe einer empirischen Studie die Ideologie zu rekonstruieren. 
In unserem Beitrag widmen wir uns zunächst dem Zusammenhang von Digitalisierung, Kapitalismus und Ideologie und stellen den Solutionismus als die Ideologie der digitalisierten Gesellschaft vor (2). Anschließend diskutieren wir das Phänomen der Sharing Economy (3). Wir stellen dann das methodische Vorgehen vor, um die Ideologie der Sharing Economy aus ihren Manifesten herauszuarbeiten (4). Im anschließenden Ergebnisteil rekonstruieren wir die Ideologiearchitektur (5), bevor wir schließlich das Fazit und einen Ausblick hinsichtlich der Ideologiekritik geben (6).

\section{Digitaler Kapitalismus, Ideologie, Solutionismus}

Die Digitalisierung nahezu aller Lebensbereiche greift immer weiter um sich und verändert unsere Lebenswelt nachhaltig. So hat sie beispielsweise neue Formen der Ökonomie angestoßen wie Microwork, Gigwork (Altenried 2017; Srnicek 2017) oder die Sharing Economy (Nachtwey und Seidl 2017). Auf der Digitalisierung liegen seit der Finanz- bzw. Vielfachkrise in den Jahren 2007/08 große Hoffnungen, auch weiterhin wirtschaftliches Wachstum zu stimulieren und bestimmte Krisenerscheinungen abzumildern. Ungebrochen scheint hier das Fortschrittsnarrativ eines nunmehr "digitalen Kapitalismus“ (Staab und Nachtwey 2016). Die Grundlagen des digitalen Kapitalismus und seine zugrundeliegende Ideologie sollen an dieser Stelle kurz beleuchtet werden.

Ideologie verstehen wir hier mit Mannheim (1985) als ein Denkmodell, das die eigene Weltsicht als objektiv, frei von Partikularinteressen und damit als generell gültig darstellt. Dabei werden bestimmte Grundannahmen nicht nur nicht reflektiert, sondern verschwinden aus dem Blickfeld: Aus dem Anspruch der Ideologie auf generelle Gültigkeit resultiert eine Verabsolutierung der eigenen Position und eine Immunisierung gegen Kritik. Ideologien vernichten somit Kontingenz. Wir bezeichnen diese Kontingenzvernichtung als "Invisibilisierung“. Invisibilisierungen führen dazu, dass Annahmen nicht weiter begründungspflichtig sind. Daraus folgt aber gleichzeitig, dass die Identifikation von Invisibilisierungen und das damit einhergehende Infragestellen der Gültigkeit ideologischer Grundannahmen einen wichtigen Ansatzpunkt für die Ideologiekritik darstellt.

Kernstück des digitalen Kapitalismus ist die „innovative Disruption“ (Nachtwey und Staab 2017, 79; Staab 2016, 42 f.) als Wachstumsprinzip. 
Innovative Disruption hebt darauf ab, neue Märkte und Geschäftsmodelle zu „erfinden“ bzw., wenn nötig, analoge Modelle zu digitalisieren. Die Digitalisierung erlaubt hierbei eine weiterführende Rationalisierung von Distribution und Konsum (Staab 2016). Die Schaffung von neuen, digitalen Märkten wäre ohne die Plattformen als Infrastruktur sowie ihre systematischen Netzwerkeffekte ${ }^{1}$ kaum denkbar. Konsequenzen hat die Bewegung hin zu digitalen Winner-takes-it-all-Märkten und in Richtung von "natürlichen Monopolen“ vor allem für die Arbeiter*innen in Gestalt einer immer stärker zerklüfteten Arbeitsorganisation (bspw. als Gigwork, auch auf mehreren Plattformen) ohne organisationale Anbindung (als Solo-Selbstständige/Freelancer). Die Arbeiter*innen begegnen sich als freie Marktakteur*innen, die miteinander um Aufträge konkurrieren müssen - und dies ohne soziale Absicherung.

Ideologische Grundlage des digitalen Kapitalismus bildet jene Kombination aus liberalem Individualismus, in Gestalt des individualisierten Unternehmers auf einem möglichst freien Markt, und technischem Determinismus, die sich als ein Geist des Solutionismus beschreiben lässt: „die Idee (...), zahlreiche Probleme der Menschheit in Form ertragreicher Geschäftsmodelle einer technologischen Lösung zuführen zu können" (Staab 2016, 39). Bridle $(2018,4)$ spitzt den so benannten Geist weiter zu und ergänzt ihn um die Vorstellung des computational thinking als "the belief that any given problem can be solved by application of computation. Whatever the practical or social problem we face, there is an app for it." Computational thinking meint hierbei eine Fortführung bzw. Radikalisierung des solutionistischen Geistes in der Form, dass ein Denken außerhalb des rechengestützten Denkens beinahe unmöglich erscheint.

Grundlegend für diesen Geist ist die namensgebende Solution, also die Lösung von bestimmten (Menschheits-)Problemen. Ihren Ursprung hat diese Lösung in der digitalen Welt, die als Denkschablone für die Problemlösungen dient (Nachtwey \& Seidl 2017, 21). Das Internet als der Ausdruck des Digitalen schlechthin ist hierbei sowohl Katalysator im Sinne von Lösungsansätzen und damit Geschäftsmodellen wie auch Rechtfertigung qua bisherigen Erfolgs (etwa Lang 2017). Dem liegt die

1 Diese Netzwerkeffekte lassen als systematische Matthäus-Effekte diejenigen Plattformen bis zu einem bestimmten Maße weiterwachsen, die bereits über eine große Nutzerzahl verfügen, da hier über die User überhaupt erst das Angebot entsteht. 
vereinfachende Annahme zugrunde, dass die zu lösenden gesellschaftlichen Probleme (1) überhaupt und (2) ausschließlich als technologische Probleme definierbar sind. Hier wird ein Steuerungsanspruch erkennbar, der wiederum jenseits von politischen oder gesellschaftlichen Aushandlungen liegt bzw. diese delegitimiert.

Zweite Grundsäule neben dem Technikdeterminismus dieses Geistes ist das Unternehmertum, von Nachtwey \& Seidl (2017) als „Weltverbesserungsunternehmerin“ auf den Punkt gebracht. Die Weltverbesserungsunternehmerin vereint eine Überhöhung der Innovation mit einem radikal-liberalen Individualismus, in dem alle Menschen als Marktteilnehmer*innen auf sich allein gestellt sind. Diese Vorstellung mündet in einen rigorosen Anti-Institutionalismus (Staab 2016, 47 f.). Institutionen gelten aus dieser ideologischen Perspektive als Innovationshemmnis schlechthin. Diese Abwertung betrifft neben Regulationsinstitutionen auch Interessensvertretungen wie Gewerkschaften, die ganz im Sinne des (Neo-)Liberalismus als Kollektive abgelehnt werden. Die Weltverbesserungsunternehmerin adaptiert eine Ingenieursperspektive, die die Lösung von Problemen mit eben jenen „rationalen“ und technologischen Mitteln vorsieht, die neben Innovation und Gewinn keine anderen Handlungsmaximen wie Gemeinwohlorientierung oder kollektive Entscheidungsfindungen kennen. Im Glauben an die „rationale“ Logik von Märkten begegnen sich alle Menschen auf diesen als freie, individualisierte Marktteilnehmer*innen.

Für unsere Zwecke zusammengefasst lässt sich der Solutionismus über die folgenden Charakteristika bestimmen: einen ungebrochenen Fortschrittsglauben (Welt verbessern, Probleme lösen), seinem Antagonismus zur "old economy" mit ihren innovationshemmenden Institutionen (ethische Bedenken, politische Aushandlung, Regulation) sowie der Allianz aus Technikdeterminismus (soziale als technologisch zu lösende Probleme) und Unternehmertum (als Avantgarde).

\section{Die Sharing Economy}

Die Sharing Economy ist ein herausstechendes Beispiel innerhalb des digitalen Kapitalismus (Srnicek 2017). Daher gehen wir davon aus, dass sich seine Ideologie des Solutionismus am Beispiel der Sharing Economy besonders eindrücklich nachvollziehen lässt. 
Die Sharing Economy ist ein heterogenes Feld, das diverse Praktiken, Austauschbeziehungen und Geschäftsmodelle unter einem begrifflichen Konstrukt vereint. Die Spannweite reicht dabei von Community-zentrierten und lokal begrenzten Plattformen wie Tauschgarten bis hin zu weltweit operierenden, Venture-Capital-finanzierten Giganten wie Airbnb und Uber.

Hertwig und Papsdorf $(2017,524)$, die aus einem Panorama an Definitionsvorschlägen (etwa Acquier, Daudigeos und Pinkse 2017) einen Minimalkonsens ableiten, verstehen die Sharing Economy als „ein Set von Geschäftsmodellen, Plattformen und Austauschbeziehungen (...), in dem unter der Beteiligung von Privatpersonen (Peer-to-Peer) Ressourcen, Dienstleistungen oder das Zugangsrecht zu Dingen (temporär) gegen eine Gebühr oder andere Kompensation (...) über Social-Media-Plattformen des Internets (...) verschenkt, getauscht, geliehen oder verkauft werden“" Hervorzuheben sind hier drei Aspekte: (1) Privatpersonen sind beteiligt, d. h. der peer-to-peer-Gedanke ist konstitutiv. (2) Der Sharing-Begriff wird weit ausgelegt und beschränkt sich nicht auf Teilen im engeren Sinne („collaborative consumption“), sondern umfasst ebenfalls "traditional sharing, bartering, lending, trading, gifting and swapping" (Botsman und Rogers 2011, XV). Aus kritischer Perspektive wird diese Ausweitung des Sharing-Begriffs jenseits der ursprünglichen Bedeutung als "Sharewashing" bezeichnet (Belk 2014), weil hier bewusst die Nähe zum prosozialen Teilen für im Grunde kommerzielle Praktiken gesucht wird. (3) Sharing-Transaktionen erfolgen über Plattformen, die als OnlineMarktplätze fungieren (Einav, Farronato und Levin 2016).

Sharing-Plattformen erhalten ihre zentrale Stellung in der Sharing Economy dadurch, dass sie als Marktplätze und zugleich als Organisationsprinzip schlechthin in erheblichem Maße für die Skalierung und damit auch den Erfolg (bzw. das Wachstum) der Sharing Economy verantwortlich sind. Sie ermöglichen darüber hinaus das "stranger sharing" (Schor 2014), also Sharing-Transaktionen zwischen zuvor nicht manifest verbundenen Knoten im Netzwerk, für beinahe jedermann, weil sie die Teilnahmehürden niedrig halten (in der Regel reicht das gewünschte Objekt zum Tausch bzw. die materiellen Voraussetzungen sowie ein Internetzugang aus).

Weiterhin unterscheiden Hertwig und Papsdorf (2017) in ihrer Studie zwischen drei sich zum Teil überlappenden Segmenten der Sharing Eco- 
nomy: (1) die Tausch- und Schenkwirtschaft, (2) die Nischen- und Nebenerwerbswirtschaft sowie (3) die Plattform-Ökonomie. Die Tausch- und Schenkwirtschaft entspricht am weitesten dem ursprünglichen Gedanken vom Teilen, weil hier oftmals güterspezifische Tauschbeziehungen bestehen, die weder von einer Äquivalenz der getauschten Güter ausgehen noch eine monetäre Kompensation beinhalten. Mit der Nischen- und Nebenerwerbswirtschaft ist hingegen das Segment bezeichnet, in dem Nutzer*innen vorrangig noch nicht kommerzialisierte Dienstleistungen anbieten (Hertwig und Papsdorf 2017). Die Plattform-Ökonomie ist laut Hertwig und Papsdorf schließlich nur durch Selbstzuschreibung qua Nutzung der Plattformen als Teil der Sharing Economy zu bezeichnen, weil hier ein sehr weiter Sharing-Begriff die Tätigkeiten und angebotenen Dienstleistungen verbindet. Gewissermaßen finden sich hier oftmals „plattformisierte“ Angebote und Dienstleistungen der bestehenden Ökonomie („old economy“), z. B. Übersetzungen oder das Erstellen von Werbetexten. Diese auch vormals existierenden Angebote werden unter Rekurs auf die Sharing-Rhetorik und qua Plattformeinsatz ins Digitale und damit auf digitale Märkte überführt.

\section{Methode und Datenkorpus}

Ziel des Beitrages ist es, die Ideologiearchitektur der Sharing Economy zu rekonstruieren. Als Ideologiearchitekur verstehen wir die einzelnen Elemente (z. B. Ausgangsprobleme, Versprechen, vorgeschlagene Lösungen), aus denen sich eine Ideologie zusammensetzt, sowie die Verbindungen dieser Elemente. Um diese Architektur zu rekonstruieren, analysieren wir grundlegende Manifeste und Anti-Manifeste der Sharing Economy mit Hilfe der narrativen Diskursanalyse (NDA) (Viehöver 2000; Viehöver 2001; Viehöver 2004).

\subsection{Sampling und Datenkorpus}

Als Sharing-Economy-Manifeste verstehen wir Werke, die (1) eine Vision der Sharing Economy entwickeln. Diese Manifeste zielen (2) auf den öffentlichen Diskurs und auf ein möglichst breites Publikum abseits von spezialisierten Handlungsbereichen ab (bspw. Wissenschaft oder Politik). 
Schließlich entwerfen sie (3) ein positives, d. h. gesellschaftlich wünschenswertes Zukunftsszenario. Wir gehen davon aus, dass sich die Ideologie der Sharing Economy in ihren Manifesten erkennen lässt.

Zusätzlich analysieren wir Anti-Manifeste. Diese Werke erfüllen wie die Manifeste die Bestimmungskriterien (1) und (2), allerdings entwerfen Anti-Manifeste (3) ein negatives Zukunftsszenario. Die Kontrastierung der Manifeste mit den Anti-Manifesten soll es uns erlauben, im Modus der Beobachtung zweiter Ordnung (von Foerster 1984) die ideologischen Invisibilisierungen zu erkennen. Tabelle 1 und 2 geben einen Überblick über die analysierten Manifeste und Anti-Manifeste.

Tab. 1: Analysierte Manifeste der Sharing Economy

\begin{tabular}{|l|l|l|l|}
\hline Nr. & Autor*innen & Jahr & Titel \\
\hline M1 & $\begin{array}{l}\text { Botsman \& } \\
\text { Roger }\end{array}$ & 2011 & $\begin{array}{l}\text { What's mine is yours: How collabora- } \\
\text { tive consumption is challenging the } \\
\text { way we live }\end{array}$ \\
\hline M2 & Buczynski & 2013 & $\begin{array}{l}\text { Sharing is good: How to save money, } \\
\text { time and resources through collabora- } \\
\text { tive consumption }\end{array}$ \\
\hline M3 & Chase & 2015 & $\begin{array}{l}\text { Peers Inc.: How people and platforms } \\
\text { are inventing the collaborative econ- } \\
\text { omy and reinventing capitalism }\end{array}$ \\
\hline M4 & Gansky & 2010 & $\begin{array}{l}\text { The mesh: Why the future of business } \\
\text { is sharing }\end{array}$ \\
\hline M5 & Glenn & 2016 & $\begin{array}{l}\text { Secrets of the sharing economy: Unof- } \\
\text { ficial guide to using Airbnb, Uber, \& } \\
\text { more to earn \$1000's }\end{array}$ \\
\hline M6 & Graham & 2017 & $\begin{array}{l}\text { The Uber of everything: How the } \\
\text { freed market economy is disrupting } \\
\text { and delighting }\end{array}$ \\
\hline M7 & $\begin{array}{l}\text { Harris \& } \\
\text { Gorenflo }\end{array}$ & 2012 & $\begin{array}{l}\text { Share or die: Voices of the get lost } \\
\text { generation in the age of crisis }\end{array}$ \\
\hline
\end{tabular}




\begin{tabular}{|l|l|l|l|}
\hline M8 & Rifkin & 2014 & $\begin{array}{l}\text { The marginal cost society: The inter- } \\
\text { net of things, the collaborative com- } \\
\text { mons, and the eclipse of capitalism }\end{array}$ \\
\hline M9 & $\begin{array}{l}\text { Rustrum, } \\
\text { Stempinski, } \\
\text { \& Liss }\end{array}$ & 2014 & $\begin{array}{l}\text { It's a shareable life: A practical guide } \\
\text { to sharing. }\end{array}$ \\
\hline M10 & Stephany & 2015 & $\begin{array}{l}\text { The business of sharing: Making it in } \\
\text { the new sharing economy }\end{array}$ \\
\hline
\end{tabular}

Tab. 2: Analysierte Anti-Manifeste der Sharing Economy

\begin{tabular}{|l|l|l|l|}
\hline Nr. & Autor*innen & Jahr & Titel \\
\hline A1 & Hill & 2015 & $\begin{array}{l}\text { Raw deal: How the 'Uber economy' } \\
\text { and runaway capitalism are screwing } \\
\text { American workers }\end{array}$ \\
\hline A2 & Scholz & 2016 & $\begin{array}{l}\text { Uberworked and underpaid: How } \\
\text { workers are disrupting the digital } \\
\text { economy }\end{array}$ \\
\hline A3 & Slee & 2015 & $\begin{array}{l}\text { What's yours is mine: Against the } \\
\text { sharing economy }\end{array}$ \\
\hline
\end{tabular}

Das Sampling erfolgte in Form des Theoretischen Samplings. Ausgangspunkt stellte Botsman und Rogers „What's Mine Is Yours“ (2011) dar. Wir haben es als Ausgangspunkt gewählt, weil dieses Werk als die "Sharing-

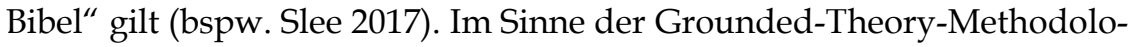
gie haben wir in einem kontinuierlichen Prozess zwischen Datensammlung und Analyse des Datenkorpus im Rahmen unserer NDA die Auswertung vorgenommen. Diesen Prozess haben wir so lange fortgeführt, bis wir eine theoretische Sättigung erreicht haben.

\subsection{Datenauswertung}

Wie van Dijk $(2006,115)$ herausstellt, eignet sich die kritische Diskursanalyse zur Untersuchung von Ideologien, denn „people acquire, express and 
reproduce their ideologies largely by text or talk". Wir betrachten die Manifeste als Konkretisierungen der (latenten) Ideologie in einzelnen Narrationen. Damit ist für die kritische Betrachtung von Ideologien bedeutsam, dass sich aus dem Diskurs um die Sharing Economy die Ideologiearchitektur und -fragmente rekonstruieren lassen. Wir gehen davon aus, dass nicht in jedem Manifest die Ideologie im vollen Umfang zu finden sein wird bzw. dass graduelle Unterschiede auftreten können (Ideologiefragmente). Mit anderen Worten: Wir verstehen die einzelnen Manifeste als Variationen über die latente Ideologie. Daher, so unsere Annahme, wird die Ideologie der Sharing Economy erst in der Gesamtschau über die verschiedenen Manifeste und in Kontrastierung mit den Anti-Manifesten in vollem Umfang greifbar.

Die von uns in dieser Studie genutzte NDA ist der genealogisch-kritischen Strömung der Diskursanalyse zuzuordnen (Heindl 2015). Sie zielt darauf $a b$, die ideologischen Grundlagen von öffentlichen Narrativen und Diskursen zu analysieren. Schlüssel zum Verständnis der potenziellen Wirkungsmacht ist hierbei die narrative Struktur der Ideologiearchitektur. Diese narrative Struktur besteht aus mehreren Kernelementen: (1) Das Narrativ beginnt mit einem oder mehreren Anfangsproblemen, die es als Ausgangspunkt setzt. (2) Es schlägt eine Lösung für das Problem bzw. die Probleme vor, die es (3) an Akteure bindet, die die Lösung durch ihre Handlungen entweder herbeiführen oder aber verhindern können. (4) Aus diesen Strukturelemente lässt sich die Storyline rekonstruieren, die Probleme, Lösungen und Akteure miteinander in Verbindung setzt. 


\section{Die Ideologie der Sharing Economy}

Die Ideologiearchitektur der Sharing Economy lässt sich aus den Manifesten und Antimanifesten wie im Folgenden dargestellt rekonstruieren (s. Abb. 1).

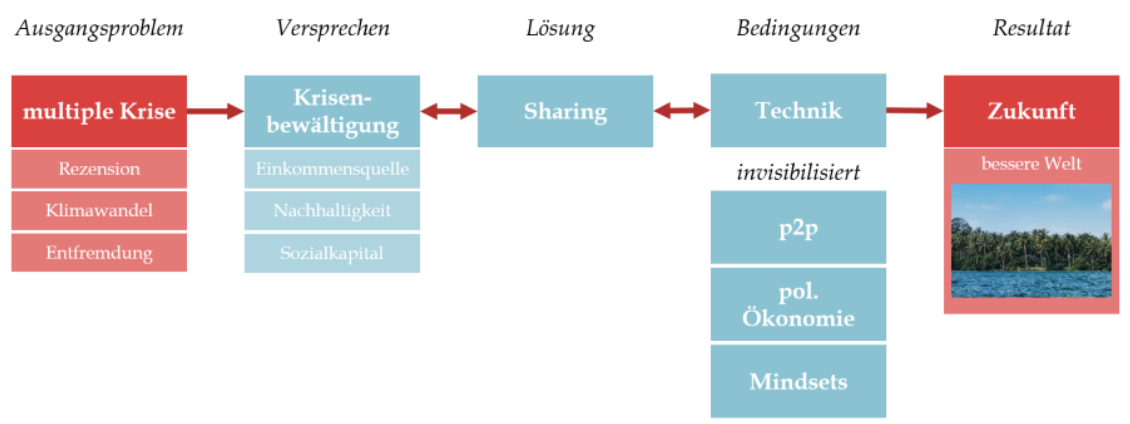

Abb. 1: Die Ideologiearchitektur der Sharing Economy

\subsection{Ausgangsproblem}

Zunächst ist mit der multiplen Krise ein Startproblem benannt, das sich analytisch in drei Dimensionen unterteilen lässt. Die erste Dimension bzw. Krise ist die im Nachgang der Finanzkrise 2007 einsetzende Rezession und die damit verbundene Schwächung der gesamten Wirtschaft. Von dieser sind sowohl einfache (potenzielle) User*innen der Sharing Economy betroffen, deren Einkommensmöglichkeiten und Haushaltsbudgets schwinden, wie auch Unternehmer*innen und Investor*innen, die nach Geschäfts- und Investitionsmöglichkeiten suchen. Damit in Verbindung steht ein zunehmendes Bewusstsein für Umweltprobleme, die zu einer Klimakrise führen. Angetrieben werden diese Umweltprobleme von der in der modernen, kapitalistischen Gesellschaft forcierten Konsumkultur eines exzessiven Konsumismus: „Changing all our light bulbs does not absolve us of a lifetime of rampant comsuption. It won't change the fact that we're running out of food, water, and clean air in an increasingly crowded planet" (Buczynski 2013,37). Angesprochen ist hier ebenfalls die dritte Dimension, die auf die zunehmende Verdichtung der Städte (Urbanisierung) bei gleichzeitigem Verlust sozialer Bindungen und 
Entfremdung hinweist, die durch das anonyme Leben im urbanen Raum zunehmend droht. Diese Krisen werden jeweils unterschiedlich stark betont, bleiben aber grundsätzlich Ausgangspunkt der Narrationen.

\subsection{Versprechen}

Die Sharing Economy verspricht, diese Probleme zu lösen bzw. zumindest ihre Auswirkungen abzumildern. Sie ist damit sowohl Antwort auf die aufgeworfenen Probleme als auch deren geradezu direkte Konsequenz. $\mathrm{Zu}$ ihren Versprechen gehören an erster Stelle die ökonomischen Möglichkeiten, die sich aus dem durch die Sharing Economy ermöglichten Sharing-Aktivitäten ergeben. Unterscheiden lassen sich hier zwei Perspektiven. Erstens werden innerhalb des Narrativs die (potenziellen) Nutzer*innen adressiert, da die Sharing Economy ihnen ein Nebeneinkommen ermöglicht, um einerseits ihre Einkommensquellen zu diversifizieren (d. h. nicht nur abhängig von einem Arbeitgeber zu sein) und andererseits die Kosten für den Zugang zu Dienstleistungen und Gütern zu senken. Ideologiekonform sind die Nutzer*innen als „micro-entrepreneurs" selbstbestimmt und flexibel, sich wahlweise auf andere Aktivitäten zu konzentrieren oder etwa andere Lebensbereiche wie die Familie zu fokussieren: „[S]he decided to furnish the spare room and rent it out on Airbnb for about fifteen nights per month. 'I can control when I'm sharing the space and when I'm not,' she explained. 'I use the extra cash to help payoff my credit card debt, and it covers about half the rent'" (Botsman und Rogers 2011, xii).

Wichtig für die Kohärenz und ideologische Stimmigkeit ist die Charakterisierung der Sharing Economy als Peer-Economy. Erstens werden die Nutzer*innen als gleichberechtigte Teilnehmer*innen gedacht. Zweitens wird den Sharing-Transaktionen eine Reziprozitätserwartung als konstitutiv eingeschrieben, d. h. die Rollen der Anbietenden und der Nachfragenden sind wechselbar und sollten prinzipiell auch gewechselt werden. Drittens verspricht die peer-to-peer-Konstellation die Möglichkeit, Intermediäre zu umgehen, so direkt mit den Transaktionspartner*innen $\mathrm{zu}$ interagieren und gleichzeitig die Transaktionskosten zu senken. Aus dieser Konzeption als Peer-Economy ergibt sich also ein explizites Ermächtigungspotenzial, das Nutzer*innen durch ihre Teilnahme an Sharing-Transaktionen realisieren können. 
Weitere Adressaten sind potenzielle Plattform-Gründer*innen (und Investor*innen) als Träger*innen des Fortschritts, die mit entsprechend innovativen und disruptiven Sharing-Plattform-Ideen neue Investitionsfelder finden. Auch sie sind selbstverständlich Unternehmer*innen, aus deren Perspektive das Grundprinzip wie folgt zusammengefasst werden kann: "'sell' the same product multiple times. Multiple sales multiply profits, and customer contact. (...) Not always and not for everything, but a Mesh network that manages shared transactions has the growing capacity to soar past a company sells something once to one owner" (Gansky 2010, 6). Hier wird das Versprechen deutlich, dass sich jedes teilbare Produkt/Dienstleistung mit Hilfe der Plattformen als Koordinationsprinzip zu einem skalierbaren Unternehmen ausbauen lässt. Es zeigt sich die oftmals implizite Orientierung an einer Unterstützung durch Venture Capital, ohne die die notwendige Skalierung nur schwer möglich ist.

Das zweite Versprechen besteht darin, dass die Sharing Economy eine nachhaltigere Alternative zum aktuellen Wirtschaften bietet. Ihre Nachhaltigkeit beruht maßgeblich auf einer effizienteren Nutzung bzw. Verteilung von existierenden Ressourcen. Ikonisches und vielfach bemühtes Beispiel ist die Bohrmaschine, die den größten Teil ihres Daseins ungenutzt fristet und nur für einen Bruchteil ihrer Lebensdauer genutzt wird. Sharing-Plattformen ermöglichen eine effizientere Nutzung, indem sie qua ihrer technologischen Infrastruktur das Teilen mit Fremden deutlich erleichtern und zugleich den Wandel zu einem zugangsbasierten Wirtschaften unterstützen. Weil das Sharing in den meisten Fällen eine kostengünstigere Option darstellt als die eigene Anschaffung, ergibt sich, so die Hoffnung, nachhaltiges Handeln als erwünschtes Nebenprodukt, ohne dass Nutzer*innen bewusst "grün" handeln müssten.

Dem dritten Problem, den dysfunktionalen Folgen der Urbanisierung, wird durch die effizientere Nutzung von Leerlaufkapazitäten (,under-utilized assets“) begegnet. Diese effizientere Nutzung wirkt, wie in dem zweiten Versprechen verdeutlicht, zugleich dem immanenten Problem der Verdichtung innerhalb von Städten entgegen und gewährleistet die effizientere Verteilung von Ressourcen. Darüber hinaus wird eine Stärkung von sozialen Beziehungen (i. S. v. sozialem Kapital) durch die Sharing Economy proklamiert: „Unlike digital forms of sharing such as poking, liking, and tweeting, the sharing economy gives people the ability to help one another get their needs met offline, oftentimes through faceto-face interactions, which many studies show, people are starved for." 
(Rustrum, Stempinski \& Liss 2014, 26) Gleichzeitig entsteht durch Plattform-vermittelte Sharing-Transaktionen das notwendige Vertrauen, durch das überhaupt erst Tauschprozesse unter Fremden in Gang gesetzt werden. Dieses Vertrauen ist nicht auf die Transaktionsprozesse und die Sharing Economy begrenzt, sondern wird als neu entstehendes, generelles gesellschaftliches Vertrauen ausgeflaggt.

\subsection{Lösung}

Die ideologisch kohärente Lösung für die Probleme ist einfach: mehr teilen bzw. gemeinsam konsumieren. Diese Lösung stellt das bisherige Konsummodell in Frage, aber nicht den Konsum an sich. Um die Lösung durchzusetzen, ist es notwendig, die Logik der Sharing-Plattformen als Geschäftsmodell auf weitere Bereiche auszuweiten, d. h. weitere lebensweltliche Aktivitäten zu identifizieren, die sich als bezahlbare Leistungen über das Plattformprinzip kommodifizieren ließen. Maßgeblich ist hierfür gerade ein Teilen im weiteren Sinne in Form von Collaborative Consumption, da sich so diverse Geschäftsmodelle und Plattformen unter der Sharing Economy vereinen lassen.

Sharing-Plattformen als Manifestationen der Technologie übernehmen die Aufgabe, Vertrauen zwischen den Transaktionspartner*innen aufzubauen, indem sie Reputationssysteme (Bewertungen, Kommentare usw.) bereitstellen. Den Plattformen wird ideologisch der Status der neutralen Infrastruktur zugeschrieben, die lediglich durch Gründer*innen und mit Hilfe von Investor*innen bereitgestellt werden muss. Die Infrastruktur reicht gemäß der Ideologie bereits aus, damit alle beteiligten Akteur*innen von den positiven Auswirkungen der Sharing Economy profitieren. Gleichzeitig sind es die Plattformen, auf denen die Innovationskraft der Sharing Economy beruht, da nur über sie und die durch sie ermöglichte Kommodifizierung von Aktivitäten überhaupt eine Skalierung erlaubt.

Neben der Koordinationsaufgabe stellen die Plattformen Marktplätze dar, auf denen sich Angebot und Nachfrage treffen sollen. Aus dem "Ausschalten“ der Intermediäre („middlemen“) ergibt sich ein weiterer Vorteil für die Nutzer*innen, denn sie können direkt miteinander handeln (im idealtypischen Sinne eines freien Marktes). Dazu bieten die Plattformen als Märkte unterstützende Funktionen wie Preisempfehlungen oder Bewertungen an. 
Auf Ebene der narrativen Struktur wird innerhalb des Diskurses ideologiebedingt vorrangig mit jeweils positiven Beispielgeschichten gearbeitet, sowohl Geschichten für und über Nutzer*innen als auch für und über Gründer*innen. Beide Gruppen werden dabei auf den gewünschten Status der Unternehmer*innen bzw. Mikro-Unternehmer*innen (SoloSelbstständige) festgeschrieben. Als solche müssen sie Risiken eingehen, indem sie gegeneinander im Wettbewerb antreten. Dazu gehört ein entsprechendes Mindset, nach dem auch das Scheitern stets als Chance begriffen werden muss und entsprechend interpretiert wird. Schließlich, so die Logik, ist das Scheitern eine Station auf dem Weg der Optimierung von Geschäftsmodellen, mit denen die geschilderten Probleme zu lösen sind. Als Antagonist in der Erzählung tritt als kollektiver Akteur die sogenannte "old economy“ in Form etablierter Anbieter (von ÖPNV bis zu kommerziellen Geschäftsmodellen wie Autovermietungen) auf. Die Sharing Economy setzt sich von der "old economy" durch ihre Innovation und die ihr innewohnenden Problemlösungspotenziale ab.

\subsection{Offengelegte Bedingungen und invisibilisierte Bedingungen}

Den Plattformen als oben beschriebener Manifestation der Technik kommt innerhalb der Ideologie der Sharing Economy die tragende Rolle zu. Sie werden als neutral und interessenlos gedacht. Neutral sind die Plattformen, weil sie nicht abseits festgelegter und vermeintlich rationaler Wege Entscheidungen treffen können, somit gewissermaßen nicht manipulierbar sind. Sie sind interessenlos, da die Frage nach dem Besitz der Plattformen als Technikmanifestationen nicht gestellt wird und die Beteiligung der User*innen, bspw. in Form von Moderationsrechten, lediglich oberflächlich bleibt und eben nicht tiefgreifende Fragen wie kollektive Besitzstrukturen berührt. Innerhalb des Solutionismus ist die Technik das Allheilmittel, welches Problem es auch immer zu lösen gilt. Die Plattformen bedürfen aus ideologischer Perspektive keiner politischen und/oder sozialen Gestaltung, weil sie als Technik sowohl die einzige Lösung sind als auch per se als "rational“ in einem ingenieurwissenschaftlichen Sinne gelten.

Die Ideologie der Sharing Economy stellt einen entsprechenden Einsatz von Technik als die eine Bedingung dar, die erfüllt sein muss, damit die Versprechen der Sharing Economy realisiert werden. Aus der Kontrastierung der Manifeste mit ihren Gegenstücken, den Anti-Manifesten, 
lassen sich jedoch drei weitere Bedingungen identifizieren: die Peer-toPeer-Architektur von Plattformen, die politische Ökonomie der Plattformen sowie ein sich vermeintlich etablierendes Sharing-Mindset.

Ausgangspunkt der Anti-Manifeste sind die mit der gegenwärtigen Sharing Economy einhergehenden Arbeitsbedingungen. Die Anti-Manifeste gehen davon aus, dass die Sharing Economy einen großen Teil der Arbeiter*innen, die in kritischer Perspektive oftmals eben als solche und nicht als Nutzer*innen bezeichnet werden², in ähnlich ausbeuterische Geschäftsbeziehungen drängt, wie es auch in traditionellen Unternehmen der Fall ist. Thematisiert werden die unbeständigen Arbeitsbedingungen (wer bekommt den "Gig“?) und damit prekären Beschäftigungsverhältnisse (mangelnde Planbarkeit, keine Sozialversicherung), die u. a. daraus resultieren, dass die Plattformen die Anbieter*innen von Dienstleistungen als Solo-Selbstständige klassifizieren. Auf diese Weise liegt die soziale Absicherung bei den Nutzer*innen selbst. Von diesem Startpunkt aus lassen sich die Invisibilisierungen, die die Ideologie der Sharing Economy vornimmt, erkennen.

Die erste Invisibilisierung folgt aus der Setzung von Sharing-Transaktionen als Peer-to-Peer-Interaktionen. Diese Setzung ist daher wichtig für die Ideologie, weil Peer-to-peer-Kommunikation als Ermächtigungsinstrument fungiert. Ist die Sharing Economy eine Peer Economy, trägt sie geradezu automatisch zur Ermächtigung ihrer Teilnehmer*innen bei: „It [die Sharing Economy] is an ode to the individual, who when she gets access to the resources she needs to create something becomes an innovative, irreplaceable producer. Most of all, it is an expression of the hope that together we can become the collaborative generation who builds an economy that does not ask us to pit owner against laborer or producer against consumer. Instead we share everything we can, because that is the only viable path forward." (Chase 2015, 139) Sobald aber die Anbieter*innen klassische Arbeiter*innen sind, die ihre Arbeitskraft gegen ein Entgelt als Dienstleistung auf einem Markt anbieten, wird die Reziprozitätserwartung von peer-to-peer-Kommunikation ausgehebelt. Mit der Reziprozitätserwartung geht auch der emanzipatorische Anspruch verloren, weil sich die Transaktionspartner*innen nun nicht mehr unter gleichen Bedingungen begegnen. Unter diesen Voraussetzungen kann auch nicht mehr

2 Auch wenn bspw. Slee (2015) doch von Nutzer*innen spricht, dann aber immer mit Zusatz der falschen Klassifikation als Selbstständige. 
von der Entstehung von sozialem Kapital die Rede sein. Ein zweiter Hinweis auf diese Invisibilisierung ergibt sich aus der Kritik durch die AntiManifeste, dass eben nicht nur Laien als Anbieter auftreten, sondern immer wieder auch professionelle Anbieter, d. h. Unternehmen (z. B. auf Airbnb, eBay, Instacart). In anderen Fällen fungiert der Plattformbetreiber sogar gleichzeitig als einziger Anbieter (z. B. Zipcar, Nextbike). Über Sharing-Plattformen werden dann klassische business-to-consumer-Transaktionen abgewickelt, die keinen emanzipatorischen Anspruch erheben.

Die zweite Invisibilisierung betrifft die politische Ökonomie der Plattformen. Diese werden im Sinne des Solutionismus als Manifestation der Technologie nicht als politisch gestaltbar gesehen, sondern ausschließlich als infrastrukturelle Grundlage. Diese Annahme begrenzt die Mitgestaltungsmöglichkeiten der User*innen auf fest abgesteckte Bereiche wie Ratingsysteme. Damit wird das in der Ideologie angelegte Ermächtigungspotenzial der Teilnahme an Sharing-Transaktionen de facto deutlich eingeschränkt. Weiterhin thematisieren die Anti-Manifeste die Besitzverhältnisse von Plattformen und schlagen Alternativen zu hierarchisch geführten, Venture-Capital-finanzierten Besitzformen vor. Damit ist die Frage der politischen und gesellschaftlichen Gestaltung gestellt, die von den Manifesten konsequent ausgeblendet wird. Diese Frage verweist direkt auf die propagierte Möglichkeit, durch die Rolle als Anbieter*in in der Sharing Economy das Haushaltseinkommen zu erhöhen. In den Manifesten erscheinen die Nutzer*innen als eine der zentralen Gruppe von Nutznießer*innen der Sharing Economy. Doch das Ausmaß, in dem sie Sharing-Einnahmen erhalten, hängt direkt von der Gestaltung der Plattform und damit von den Besitzverhältnissen ab. Diese entscheiden darüber, wer sich welchen Teil des Mehrwerts aneignet, der in SharingTransaktionen generiert wird.

Die dritte Invisibilisierung, die durch die Kontrastierung von Manifesten mit Anti-Manifesten sichtbar wird, betrifft die Annahme der gesellschaftsweiten Etablierung eines Sharing-Mindsets. Dieses Mindset besagt, dass der Zugang zu Dingen wertvoller ist als der Besitz dieser Dinge. Die Manifeste gehen davon aus, dass dieser Einstellungswandel entweder bereits bei der Vielzahl von Konsument*innen stattgefunden hat oder dass er sich zumindest bei jüngeren Konsument*innen beobachten lässt und sich daher im Generationenübergang mittel- bis langfristig vollziehen wird. Ein solcher Wandel ist zum einen notwendig, um langfristig die 
Operationsgrundlage der Sharing Economy zu sichern. Zum anderen impliziert er den Übergang in eine reduktive Moderne (sensu Welzer 2014), die ihren Ressourcenverbrauch deutlich verringert. Aber auch die Annahme eines solchen Wandels ist kontingent. Denn ob er sich tatsächlich flächendeckend konstatieren lässt bzw. erwartbar ist, lässt sich anhand der tatsächlichen Nutzung von Sharing-Angeboten nicht feststellen. So handeln beispielsweise Zipcar-Nutzer*innen aus eigennützigen Motiven und weniger auf Grund eines rückläufigen Besitzdenkens. Daraus folgt: „Despite the claims of ecological sustainability embodied in ideas like 'access over ownership' and the re-use of excess capacity, the on-demand sector [ein bestimmtes Segment der Sharing Economy] is instead encouraging a new form of privileged consumption: 'lifestyle as a service.'” (Slee 2015, 163) Mit „Lifestyle as a service“ ist dabei neben der Kritik an ondemand vor allem die Landnahme bzw. Kommodifizierung weiterer Lebensbereiche angesprochen, denn es werden immer mehr Tätigkeiten, die früher unentgeltlich erledigt wurden, monetarisiert. Adaptiert man diese Sharing-Logik, kann man immer neue Konsumbereiche "erfinden", die hauptsächlich auf zusätzlichen Komfort der Nutzer*innen abzielen und eben nicht, wie vom Slogan ,access over ownership“ ursprünglich suggeriert, auf soziale und ökologischen Aspekte verweisen.

Dazu kommt, dass das Mindset insbesondere die urbane Mittelschicht anspricht. Das hat zwei Gründe: Erstens müssen die Nutzer*innen eine bestimmte Ressourcenausstattung (ökonomisch und kulturell) aufweisen, um überhaupt an Sharing-Transaktion teilnehmen zu können. Zweitens ist diese urbane Mittelschicht kulturell dem Sharing-Mindset (nachhaltiger Konsum, weniger Besitz) nahe und damit auch affin für die in der Sharing Economy angelegten Praktiken. Auch weisen die Anti-Manifeste darauf hin, dass unklar ist, ob Access-Modelle tatsächlich zu einer Verringerung des Ressourcenverbrauchs führen. Ein genanntes Beispiel: Verringerte Zugangshürden zu Autofahrten können dazu führen, dass verstärkt Automobile als Fortbewegungsmittel z. B. zulasten des ÖPNVs gewählt werden (Slee 2015, 53). Vor diesem Hintergrund erscheint die Annahme eines Sharing-Mindsets in einem anderen Licht, denn weder ist ein entsprechender Wandel notwendig attestierbar noch ist eindeutig, dass er die ihm zugeschriebenen positiven Effekte besitzt. 


\section{Kapitalistische Kontinuitäten}

In der Analyse wird deutlich, dass die in der Sharing Economy ideologisch überhöhte Innovation vor allem für zusätzliches Wachstum sorgen soll bzw. die Hoffnung, aber auch das Versprechen nach weiterem Wachstum mit ihr verbunden ist. Um dieses Versprechen differenzierter zu betrachten, ist es hilfreich, auf die von Hertwig und Papsdorf (2017) vorgenommene Segmentierung der Sharing Economy zurückzukommen. Tatsächlich ist die Wachstumsorientierung vor allem für das Segment der Plattform-Ökonomie relevant, da hier die Skalierung der Plattformen im Vordergrund steht, nicht zuletzt auf Grund der Venture-Capital-Unterstützung. Für die Plattformen der Schenk- und Tauschwirtschaft kann diese Wachstumsorientierung nur eingeschränkt gelten, da hier sowohl auf Grund der zu tauschenden Gegenstände als auch der fehlenden Profitorientierung dem Wachstum inhärente Grenzen gesetzt sind. Im ideologischen Sinne wird mit der Orientierung auf die Plattform-Ökonomie und das Ausweiten des Sharing-Prinzips (im weiteren Sinne), das bei genauerem Hinsehen eigentlich die Plattformlogik bezeichnet, weiterhin ein Wachstums- und Fortschrittsnarrativ verfolgt. Der proklamierte Gesinnungswandel (Tauschen statt Besitzen) wird so zweifelhaft.

Die Schenk- und Tauschwirtschaft spielt de facto nur eine kleine Rolle in der globalen Sharing Economy. Sie wird aber trotzdem auf Grund des Sharing im engeren bzw. ursprünglichen Sinne (tatsächliches Teilen) oftmals als Legitimation herangezogen, wenn auf die prosoziale Natur des Sharing-Prinzips verwiesen werden soll (Hertwig und Papsdorf 2017, 535, 542). Wiederum die Plattform-Ökonomie profitiert von dieser legitimatorischen Grundlage des ursprünglichen bzw. „authentischen“ Teilens. Diskursiv wird die analytische Trennung bewusst vermieden, da oftmals von der Sharing Economy als Ganzem gesprochen wird. Die Leistung des Solutionismus als ideologischer Grundlage besteht darin, die Plattformlogik mit der ursprünglichen, pro-sozialen Bedeutung des Teilens zu verbinden, dabei aber gleichzeitig bestimmte Aspekte wie die politischen Gestaltung der sozialen Verhältnisse zu invisibilisieren. In anderen Worten: Die Ideologie ermöglicht den Brückenschlag von Schenkund Tauschwirtschaft zur Plattform-Ökonomie, indem sie die Versprechen der Schenk- und Tauschwirtschaft auf die Plattform-Ökonomie überträgt. 
Im ungebrochenen Fortschrittsnarrativ manifestiert sich, dass der proklamierte Gesinnungswandel vom Besitzen zum Tauschen eben nicht stattfindet. Stattdessen geht es weiterhin um Wachstum und damit in kritischer Lesart eben um jene Landnahme, die nur einen Teil der Kritik am Massenkonsum in sich aufnimmt, ohne das zugrundeliegende Funktionsprinzip zu hinterfragen (sensu Boltanski und Chiapello 2003). Die Sharing Economy vermag es, die an den Kapitalismus gerichtete Kritik des Massenkonsums, der Ressourcenausbeutung sowie der sozialen Entfremdung aufzunehmen, indem sie verspricht, die kritisierten Probleme durch technologische Mittel zu lösen. Diese Lösung wird jedoch ohne konkrete politische Gestaltung gedacht, da im Solutionismus stets die technisch-rationale Gestaltung des freien Marktes für die Individuen die beste Lösung bereithält. Im Geiste des Solutionismus sind dann auch die aus der technologischen Steuerung entstandenen Probleme lediglich "Bugs“. Die sozialen Bedingungen werden ausgeblendet.

Hinzu kommt eine Ideologie des freien Marktes als konstitutives Element des Solutionismus. Aus ideologischer Perspektive sind alle betroffenen Akteur*innen Unternehmer*innen, die sich auf dem Markt treffen, der möglichst ohne illegitime Eingriffe wie bspw. staatliche Regulierung bleiben soll. In der Konzeption der Plattformen als Marktplätze spiegelt sich dieser Anspruch wieder. Hier wird - nach Möglichkeit und wenn es das eigene Geschäftsmodell nicht gefährdet - den Marktkräften die Koordination überlassen. Geschäftsmodelle werden dann v. a. dadurch gefährdet, dass sich keine ausreichende Anzahl an Anbieter*innen findet, weil diese die Leistungen erbringen, von denen die Plattformbetreiber*innen ihre Rente abschöpfen.

\section{$7 \quad$ Fazit \& Ausblick}

Das Ziel unseres Beitrags bestand darin, die ideologische Grundlage des digitalen Kapitalismus anhand des Beispiels der Sharing Economy herauszuarbeiten. Dabei hat sich gezeigt, dass durchaus Möglichkeiten zur Ideologiekritik bzw. präziser ausgedrückt: zur Kritik am solutionistischen Geist des digitalen Kapitalismus gegeben sind. In der Gegenüberstellung von Mainfesten und Anti-Manifesten wird deutlich, welche Bedingungen invisibilisiert werden, die den ideologischen Prämissen gegenüberstehen. 
So wird mit der Setzung von Technik und ihrer vermeintlichen Problemlösefähigkeit ausgeblendet, dass die Sharing Economy eben auch anders aussehen könnte, weil sie weder auf die Expansion des Geschäftsmodells inklusive Venture Capital festgeschrieben werden muss noch ihre technische Umsetzung die alleinige gestalterische Stellschraube ist.

Der Wert der Anti-Manifeste besteht somit u. a. darin, dass sie die zumeist impliziten ideologischen Annahmen der Manifeste herausfordern. Diese sind implizit, weil es in der hegemonialen Denkart des Solutionismus rational und selbstevident ist, soziale als technologisch lösbare Probleme zu verstehen, aber auch, weil bestimmte (neo-)liberale Denkkategorien, bspw. das allgegenwärtige Unternehmertum und die Verabsolutierung des „freien Marktes“, stets schon integriert sind.

In der Draufsicht zeigt sich, dass sich die ideologisch proklamierten Transformationspotenziale und -hoffnungen, zugespitzt im Motto „Teilen statt Besitzen“, vor allem an marginalisierten, nicht-kommerzialisierten Segmenten der Sharing Economy festmachen lassen, dann aber im ideologischen Narrativ ihre Gültigkeit für die gesamte Sharing Economy proklamiert wird. In der gegenwärtigen Sharing Economy lässt sich daher weniger eine Überwindung kapitalistischer Logiken als vielmehr eine Transformation von ebenjenen erkennen. So ist auch die Sharing Economy ein neuerliches Beispiel dafür, wie wandlungsfähig und persistent zugleich die Logik kapitalistischen Wirtschaftens ist: wandlungsfähig, weil, wie in der Literatur zur Landnahme (bspw. Dörre 2009) beschrieben, stets neue Lebensbereiche kommodifiziert werden (müssen); persistent, weil für die Sharing Economy dieselben Grundprinzipien gelten wie für andere kapitalistische Wirtschaftsbereiche: Gewinnmaximierung, Wachstumsabhängigkeit und Landnahme.

Was sich indes noch zeigen muss, ist, inwiefern sich mit zunehmender Kritik (1) der Diskurs verschiebt und weiterhin in der Lage ist, Kritikpunkte, wie sie etwa in den Anti-Manifesten formuliert werden, aufzunehmen, und (2) inwiefern, damit zusammenhängend, neue Akteure auf den Plan treten bzw. neue Diskurskoalitionen eingegangen werden, sodass sich die ideologische Erzählung womöglich ändert. Ein Beispiel für den Wandel von Positionen ist das Begrüßen von Regulierungen auch durch Plattformbetreiber*innen. Ziel ist es dabei oftmals, durch eigene Beteiligung den Regulierungsprozess sowohl mitzubestimmen als auch den Eingriff möglichst gering zu halten. 
Dafür schließlich, dass eine partiell andere Sharing Economy möglich ist, argumentieren insbesondere Vertreter*innen des Plattformkooperativismus (Sandoval 2016; Schneider 2018; Scholz 2017). Der Plattformkooperativismus reflektiert explizit die hier aufgezeigten Prinzipien und lehnt die in der Venture-Capital-gestützten Variante der Sharing Economy unausweichliche Gewinnabschöpfung durch Wenige und schlussendlich für Wenige ab. Er deutet so an, die ideologischen Versprechen der Sharing Economy tatsächlich erfüllen zu können. Inwieweit die Idee des Plattformkooperativismus das Potenzial besitzt, an Traktion zu gewinnen und sich sogar durchzusetzen, kann an dieser Stelle nicht beantwortet werden. Allerdings wird es dazu nötig sein, die in diesem Beitrag rekonstruierte ideologische Narration zu durchbrechen, denn der Plattformkooperativismus muss Fragen thematisieren, die sich aus einer solutionistischen Perspektive gar nicht erst stellen.

\section{Literatur}

Acquier, Aurélien, Thibault Daudigeos, und Jonatan Pinkse. 2017. „Promises and paradoxes of the sharing economy: An organizing framework". Technological Forecasting and Social Change 125:1-10. https:// doi.org/10.1016/j.techfore.2017.07.006.

Altenried, Moritz. 2017. „Die Plattform als Fabrik: Crowdwork, Digitaler Taylorismus und die Vervielfältigung von Arbeit“. Prokla 47 (187): 175-93.

Belk, Russell. 2014. „You are what you can access: Sharing and collaborative consumption online". Journal of Business Research $67 \quad$ (8): 1595-1600. https:// doi.org/10.1016/j.jbusres.2013.10.001.

Boltanski, Luc, und Ève Chiapello. 2003. Der neue Geist des Kapitalismus. Konstanz: UVK.

Botsman, Rachel, und Roo Rogers. 2011. What's mine is yours: How collaborative consumption is challenging the way we live (2. durchges. u. actual. Aufl.). London: Collins.

Bridle, James. 2018. New dark age: Technology and the end of the future. London: Verso.

Dörre, Klaus. 2009. „Die neue Landnahme. Dynamiken und Grenzen des Finanzmarktkapitalismus". In Soziologie - Kapitalismus - Kritik, hrsg. von Klaus Dörre, Stephan Lessenich und Hartmut Rosa, 21-86. Frankfurt am Main: Suhrkamp.

Einav, Liran, Chiara Farronato, and Jonathan Levin. 2016. „Peer-to-peer markets“. Annual Review of Economics 8 (1): 615-35. https://doi.org/10.1146/annurev-economics080315-015334.

Heindl, Andreas. 2015. „Diskursanalyse.“. In Methodologie, Methoden, Forschungsdesign, hrsg. von Achim Hildebrandt, Sebastian Jäckle, Frieder Wolf, und Andreas Heindl, 257-98. Wiesbaden: Springer. 
Hertwig, Markus, und Christian Papsdorf. 2017. „Varieties of sharing: Handlungsorientierungen, Strukturen und Arbeitsbedingungen eines neuartigen Feldes". Berlin Journal für Soziologie 27 (3-4): 521-46. https:// doi.org/10.1007/s11609-018-0359-5.

Lang, Susanne. 2017. „Eine kurze Geschichte des Internets: Die Inkorporation des Internets in kapitalistische Verhältnisse ist keinesfalls abgeschlossen und noch umkämpft". Prokla 47 (186): 7-27.

Mannheim, Karl. 1985. Ideologie und Utopie. 7. Aufl. Frankfurt am Main: Klostermann.

Nachtwey, Oliver, und Timo Seidl. 2017. „Die Ethik der Solution und der Geist des digitalen Kapitalismus.". $\quad$ http://www.ifs.uni-frankfurt.de/wp-content/uploads/IfS-WP11.pdf. Zugegriffen: 15. November 2019.

Nachtwey, Oliver, und Philipp Staab. 2017. „Die Avantgarde des digitalen Kapitalismus“. In Kapitalismus und Ungleichheit: Die neuen Verwerfungen, herausgegeben von Heinz Bude und Philipp Staab. Bonn: Bundeszentrale für politische Bildung.

Sandoval, Marisol. 2016. „What would Rosa do? Co-operatives and radical politics.“ Soundings, 63: 98-111. https:// doi.org/10.3898/136266216819377039

Schneider, Nathan. 2018. Everything for everyone: The radical tradition that is shaping the next economy. New York: Nation.

Scholz, Trebor. 2017. Uberworked and underpaid: How workers are disrupting the digital economy. Cambridge: Polity.

Schor, Juliet B. 2014. „Debating the Sharing Economy“. https://greattransition.org/publication/debating-the-sharing-economy. Zugegriffen: 15. November 2019.

Slee, Tom. 2017. What's yours is mine: Against the sharing economy. New York: Or.

Srnicek, Nick. 2017. Platform Capitalism. Cambridge: Polity.

Staab, Philipp. 2016. Falsche Versprechen: Wachstum im digitalen Kapitalismus. Hamburg: Hamburger Edition.

Staab, Philipp, und Oliver Nachtwey. 2016. „Market and labour control in digital capitalism“. tripleC 14 (2). https://doi.org/10.31269/triplec.v14i2.755.

van Dijk, Teun A. 2006. „Ideology and discourse analysis“. Journal of Political Ideologies 11 (2): 115-40. https:// doi.org/10.1080/13569310600687908.

Viehöver, Willy. 2000. „Political negotiation and co-operation in the shadow of public discourse: the formation of the German waste management system DSD as a case study“. European Environment 10: 277-92.

Viehöver, Willy. 2001. „Diskurse als Narrationen“. In Handbuch Sozialwissenschaftliche Diskursanalyse, Bd. 1: Theorien und Methoden, hrsg. von Reiner Keller, Andreas Hirseland, Werner Schneider, und Willy Viehöver, 177-206. Wiesbaden: VS.

Viehöver, Willy. 2004. „Die Wissenschaft und die Wiederverzauberung des sublunaren Raumes. Der Klimadiskurs im Licht der narrativen Diskursanalyse“. In Handbuch Sozialwissenschaftliche Diskursanalyse, Bd. 2: Forschungspraxis, hrsg. von Reiner Keller, Andreas Hirseland, Werner Schneider, und Willy Viehöver, 233-269. Wiesbaden: VS.

von Foerster, Heinz. 1984. Observing systems. Seaside: Intersystems.

Welzer, Harald. 2014. Selbst denken: Eine Anleitung zum Widerstand. Frankfurt am Main: Fischer. 


\section{Open Access}

Dieser Beitrag erscheint unter der Creative-Commons-Lizenz CC-BY-SA 4.0: https://creativecommons.org/licenses/by-sa/4.0/ 